Trauma Berufskrankh 2012 · 14 [Suppl 4]:411 DOI 10.1007/s10039-012-1866-9

Online publiziert: 15. November 2012

๑) Springer-Verlag Berlin Heidelberg 2012

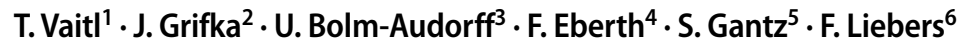

M. Schiltenwolf ${ }^{5} \cdot$ G. Spahn ${ }^{7}$

${ }^{1}$ Regensburger OrthopädenGemeinschaft, Regenstauf

2 Orthopädische Klinik für die Universität Regensburg, Bad Abbach

${ }^{3}$ Abteilung Arbeitsschutz und Umwelt, Dezernat Landesgewerbearzt, Regierungspräsidium Darmstadt, Wiesbaden

${ }^{4}$ Landesamt für Arbeitsschutz Brandenburg, Potsdam

${ }^{5}$ Department Orthopädie, Unfallchirurgie und Paraplegiologie, Universitätsklinikum Heidelberg

${ }^{6}$ Bundesanstalt für Arbeitsschutz und Arbeitsmedizin (BAuA), Berlin

${ }^{7}$ Praxisklinik für Unfallchirurgie und Orthopädie Eisenach

\title{
Infekte im Knie
}

\section{Hintergrund}

DOI 10.1007/s10039-012-1866-9

(c) Springer-Verlag Berlin Heidelberg 2012

T. Vaitl · J. Grifka · U. Bolm-Audorff

F. Eberth · S. Gantz · F. Liebers

M. Schiltenwolf $\cdot$ G. Spahn

Infekte im Knie

\section{Zusammenfassung}

Hintergrund. Haben keimbedingte Knieinfektionen Einfluss auf die Entstehung einer

Gonarthrose?

Methodik. Pubmed-Literaturrecherche Ergebnisse. Kein Nachweis von Längsschnittstudie, aber Hinweise auf schwere Verläufe.

Schlussfolgerung. Trotz fehlender Studienlage können laut Expertenmeinung gravierende Infektionen zu einer Gonarthrose führen.

\section{Schlüsselwörter}

Knie · Infektion · Keim · Gonarthrose .

Literaturrecherche

\section{Knee infections}

\section{Abstract}

Background. Do bacterial infections cause osteoarthritis of the knee?

Methods. PubMed literature search Results. No longitudinal studies could be found, but some evidence of severe progression.

Conclusion. The expert opinion is that severe infections can lead to osteoarthritis of the knee, despite the lack of studies.

\section{Keywords}

Knee · Infection · Bacteria · Osteoarthritis, knee $\cdot$ Literature
Unter Kniegelenkinfektionen werden im vorliegenden Beitrag keimbedingte Infektionen subsumiert.

Bei keimbedingten Infektionen mit den typischen Merkmalen (Rötung, Schwellung, Schmerz, Erwärmung und Funktionsminderung) kann es zur Eiterbildung im Gelenk kommen (Kniegelenkempyem). Dieser Zustand erfordert eine chirurgische Intervention im Sinne einer Spülung und Schleimhautentfernung des Kniegelenkes.

\section{Methodik}

Es wurde eine Pubmed-Recherche bezüglich der Entstehung einer Arthrose nach einem bakteriellen Infekt durchgeführt. Folgende Suchbegriffe wurden verwendet:

- „infection“ AND „osteoarthritis knee“ (741 Suchergebnisse),

- "secondary osteoarthitis knee“ (776 Suchergebnisse),

- „inflammation“ AND „osteoasthritis knee“ (639 Suchergebnisse).

\section{Ergebnisse}

Bei der Pubmed-Recherche konnten keine Studien gefunden werden, welche im Sinne einer Längsschnittstudie eine Korrelation zwischen Infekt und Gonarthrose sehen. In einer prospektiven Studie mit 154 Patienten zur allgemeinen Progno- se von bakteriellen Gelenkinfekten konnten bei etwa der Hälfte der Patienten nach durchschnittlich 2 Jahren schlechte Ergebnisse festgestellt werden, wobei hierunter Versteifungen, Endoprothesen, Amputationen und schlechte Funktionalität der Gelenke subsumiert wurden [1].

\section{Schlussfolgerung}

Die fehlende Studienlage spiegelt nicht das klinische Bild wider. Sehr wohl können gravierende Infektionen zu Knorpelund somit zu Gelenkveränderungen im Sinne einer Gonarthrose führen. Somit sind vorausgegangene Infekte als konkurrierender Risikofaktor zu sehen.

\section{Korrespondenzadressen}

\section{Dr. T. Vaitl}

Regensburger OrthopädenGemeinschaft, Eichendorffstraße 20, 93128 Regenstauf

\section{Prof. Dr. Dr. J. Grifka}

Orthopädische Klinik

für die Universität Regensburg,

Kaiser-Karl V.-Allee 3, 93077 Bad Abbach

Interessenkonflikt. Der korrespondierende Autor gibt für sich und seine Koautoren an, dass kein Interessenkonflikt besteht.

\section{Literatur}

1. Kaandorp CJ, Krijnen P, Moens HJ, Habbema JD, Schaardenburg D van (1997) The outcome of bacterial arthritis: a prospective community-based study. Arthritis Rheum 40(5):884-892 